\title{
Inguinal Hernia: A New (Not Anatomical) Classification
}

\author{
Alloni Rossana ${ }^{1}$, Luffarelli Paolo ${ }^{1}$, Mallozzi S. Maria Francesco ${ }^{1}$, Santoni Simone ${ }^{1}$, \\ Lichinchi Domenico Ernesto ${ }^{2}$, Vitali Massimiliano Andrea ${ }^{2}$ \\ ${ }^{1}$ General Surgery Department, Campus Bio-Medico University of Rome, Rome, Italy \\ ${ }^{2}$ Health Management Department, Campus Bio-Medico University of Rome, Rome, Italy
}

Email address:

r.alloni@unicampus.it (A. Rossana), p.luffarelli@unicampus.it (L. Paolo), francesco.mallozzi@alice.it (M. S. M. Francesco), simone.santoni1@gmail.com (S. Simone), d.lichinchi@unicampus.it (L. D. Ernesto),m.vitali@unicampus.it (V.M. Andrea)

\section{To cite this article:}

Alloni Rossana, Luffarelli Paolo, Mallozzi S. Maria Francesco, Santoni Simone, Lichinchi Domenico Ernesto, Vitali Massimiliano Andrea. Inguinal Hernia: A New (Not Anatomical) Classification. Journal of Surgery. Vol. 7, No. 3, 2019, pp. 74-77. doi: 10.11648/j.js.20190703.15

Received: April 26, 2019; Accepted: May 31, 2019; Published: June 24, 2019

\begin{abstract}
Inguinal hernia is one of the most frequently found surgical problems, accounting for about 70-75 per cent of all hernia operations. Inguinal hernia represents a social disease, with considerable management costs. All classifications of inguinal hernia have something of arbitrary and artificial, and unfortunately are based on anatomic and functional criteria. Moreover, single hernia defect can be classified only during the operation and not in a preoperative setting. The aim of this study has been to evaluate the operative times and consequently identify factors that affect the surgical time. In this way we hope to create a new classification useful to standardize the operative time management. From February 2012 to June 2013, in the Day Surgery Unit of Campus Bio-Medico University of Rome, 110 consecutive patients were enrolled which underwent to inguinal hernioplasty, and they have been observed by the same surgical team. We evaluated clinical parameters (age, sex, BMI, hernia size defect, reducibility, primitive or recurrent hernia, previous hernia surgery) and compared them with surgical times. Data analysis shows a statistically significant relationship between reducibility, recurrent hernia, male gender, BMI and surgical times. This study confirms that an optimal clinical patient evaluation should always be the first step to an effective organizational choice and it allows realistic predictions about the duration of inguinal hernioplasty.
\end{abstract}

Keywords: Inguinal Hernia, Surgical Time, Classification

\section{Introduction}

Inguinal hernia is one of the most frequently found surgical problems, accounting for about 70-75 per cent of all hernia operations [1]. Approximately, more than 20 million patients undergo inguinal hernia repair [2]. The incidence of inguinal hernia repair is 10 per 100000 population in the UK and 28 per 100000 in the USA [3].

Twenty-seven percent of men and three percent of women will develop an inguinal hernia during their lifetime. Inguinal hernia represents a social disease, with considerable management costs [4].

Worldwide men and women have a $27 \%$ and $3 \%$ risk to develop an inguinal hernia during their life [5].

According to data from the National Center for Health Statistics, recent studies have estimated between 600 and 770 surgical repairs of inguinal hernia annually and, mostly were performed on outpatients [6].

It's unknown the real recurrence rate after an inguinal hernia repair, but approximately a $10-15 \%$ of patients require a reoperation [7].

Nowadays is increasingly widespread the concept of a tailored surgical operation for each patient, including a minimal recovery, and low invasiveness, cost, recurrence rate, and post-operative pain [8].

The first inguinal hernia repair were performed at the end of the sixteenth century and it provided the reduction and resection of the hernia sac, and the reinforcement of the inguinal canal posterior wall by approximating its muscular and fascial components [2].

An important advances in this kind of surgery were introduced by Bassini (1871) and Sholudice (1950), and subsequently by Lichtenstein (1970) [9-11].

Currently, groin hernia treatment is not standardized, in 
fact in the Literature have been reported three possible choice: open, laparoscopic and robotic approach [2]. Anyway, the open approach represent the treatment of choice for the majority of surgeons, due to low costs, low length of stay (outpatients setting or day-surgery recovery), ease of execution, and minimal invasiveness.

Since 1804, inguinal hernias were traditionally classified as indirect, direct, and femoral by Cooper [12]. Since then, many classifications have been proposed. In particular, in 1987, Lichtenstein reported a 6000 cases series including his personal classification system, including three categories: indirect, direct and femoral [13]. The most recent classification was published in 1999 by Zollinger [14, 15].

Unfortunately, all classifications of inguinal hernia have something of arbitrary and artificial, and are based on anatomic and functional criteria [16]. Moreover, single hernia defect can be classified only during the operation and not in a preoperative setting.

In this study we reviewed the main classifications of inguinal hernia in scientific literature.

We looked at the issue from a different perspective: is it possible, using pre-operative evaluation of patients, to define clinical criteria that help the surgeon to estimate the complexity of the single inguinal hernia repair and consequently, times of surgery? A functional pre-operative evaluation would allow a better surgery scheduling and increase the efficiency of operating room.

The aim of this study has been to evaluate the operative times and consequently identify factors that affect the surgical time. In this way we hope to create a new classification useful to standardize the operative time management.

\section{Material and Methods}

From February 2012 to June 2013 we have consecutive enrolled 110 patients, aged between 18 and 84 years, 10 females and 100 males, affected with inguinal hernia and candidates to monolateral inguinal hernioplasty procedure.

In $98 \%$ of cases the inguinal hernioplasty was performed with a sutureless and tension free open technique. We proceed to a clinical and preoperative evaluation of each patient, according to criteria such as: age, Body Mass Index (BMI), sex, specifying whether right or left, size of the hernia (then - Width - Length - Area classifying as W1 hernias of maximum diameter less than $4 \mathrm{~cm}$, W2 hernias with a maximum diameter between 4 and $10 \mathrm{~cm}$ and $\mathrm{W} 3$ large hernias, that is, with diameter greater than $10 \mathrm{~cm}$ ), size of the hernia door in $\mathrm{cm}$, reducibility (indicating as " 1 " spontaneously reducible hernia, for example in supine position, as " 2 " hernia only manually reducible, as " 3 " an incarcerated hernia), primary or recurrent hernia, any mesh used in case of relapse, previous abdominal surgery.

To avoid bias of concordance, clinical evaluations and operation on patients, has been performed by the same surgical team, using the same surgical technique and local anesthesia. Data regarding operative time, has been collected in a database and statistical analysis to identify a possible relationship between these criteria and surgical times (from skin incision to skin closure) has been performed, either individually or in combination. Student's T test was used to evaluate the statistical significance as parametric measure of these relations.

\section{Results}

Patient's characteristics are reported in Table 1.

Table 1 Characteristics and clinical parameters of patients with inguinal hernia, that we correlated with surgical time.

Table 1. Patient's characteristics.

\begin{tabular}{ll}
\hline Characteristic & $\mathbf{n}$ \\
\hline Mean age (year) & 61 \\
Mean BMI & 25 \\
Right inguinal hernia (\%) & 65 \\
Mean width (cm) & $2.78(1.8-15)$ \\
Mean length (cm) & $3(1-20)$ \\
Average hernia door (cm) & $1.3(0.5-3)$ \\
Recurrent hernia (\%) & 12 \\
Completely reducible hernia (\%) & 89 \\
Personal history of previous surgery (\%) & 35 \\
\hline
\end{tabular}

The mean surgical time required to perform the hernioplasty procedure was 63.78 minutes, with a minimum time of 24 minutes and a maximum time of 108 minutes.

We analyzed all clinical parameters in our patients, in order to know how they affect the surgical time, as reported in Table 2.

Table 2 Parameters that affect surgical time.

Table 2. Incidence of some parameters on surgical time.

\begin{tabular}{ll}
\hline Parameter & $\mathbf{p}$ \\
\hline Reducibility & $<0.001$ \\
Recurrent hernia & $<0.05$ \\
Sex & $<0.05$ \\
BMI & $<0.001$ \\
\hline
\end{tabular}

Reducibility: completely reducible hernia and notcompletely reducible hernia. A completely reducible hernia is associated with shorter operating times than not-completely reducible hernia: 50.73 minutes (CI - confidence interval $95 \% 44.3-57.13$ minutes) in the first group versus 69.70 in the second. A statistically significant difference has been found between two groups using the Student $\mathrm{t}$ test for unpaired data $(p<0.001)$ (Figure 1). The irreducibility is therefore definitely as a variable that increases the mean surgical times.

Recurrent hernia: it needs on average $20 \pm 5$ minutes longer than the primary hernia $(p<0.05)$ (Figure 2$)$.

Sex: the female sex has been found associated with shorter mean surgical times ( -13 minutes) compared to the male sex. The difference has been statistical significant $(p<0.05)$ (Figure 3).

BMI: we have divided patients into two groups on the basis of cut-off classically used to distinguish normal weight 
subjects from the overweight subjects (BMI between 18 and $24.9=$ normal weight; BMI above $25=$ overweight). In overweight/obese patients we observed longer surgical times than in normal weight patients, quantifiable in $14 \pm 4$ minutes $(p<0.001)$ (Figure 4).

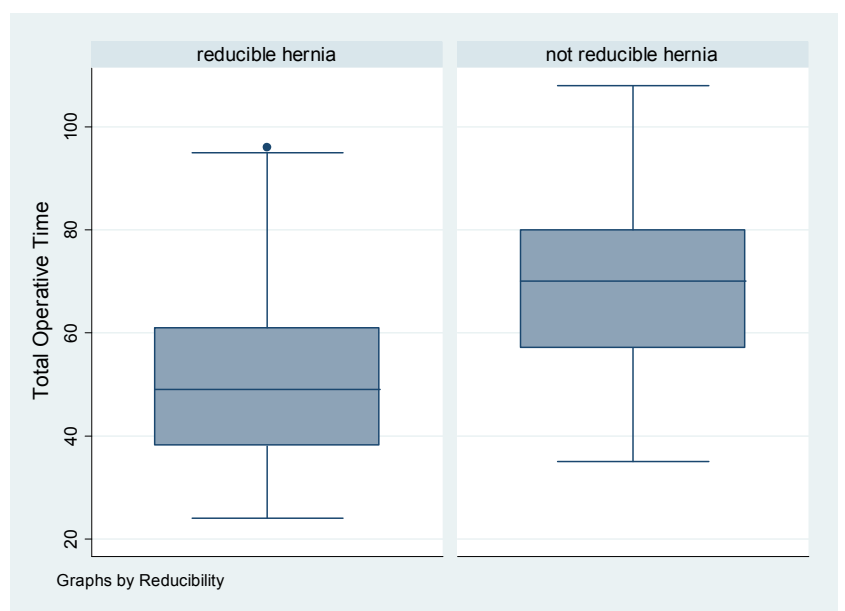

Figure 1. Variation on surgical time between reducible and not reducible inguinal hernia.

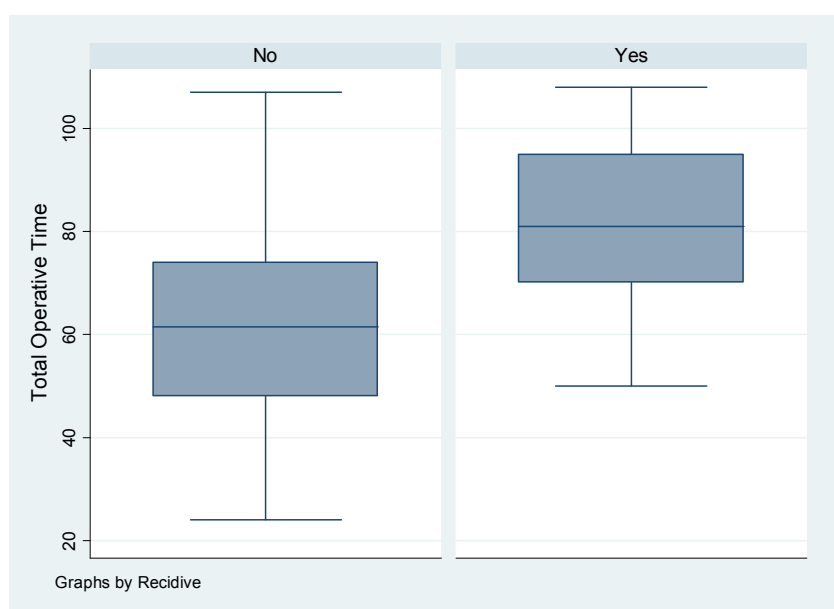

Figure 2. Variation on surgical time between primary and recurrent inguinal hernia.

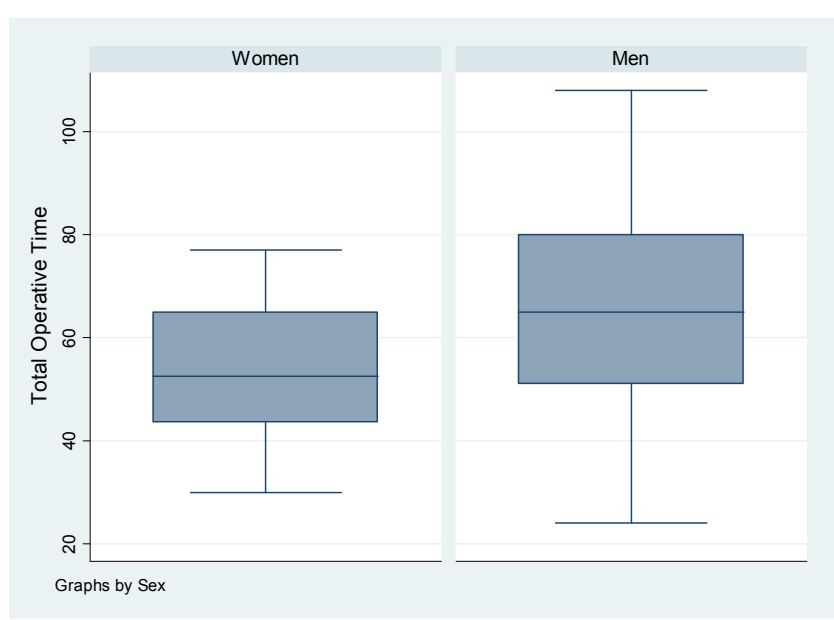

Figure 3. Variation on surgical time between male and female patients.

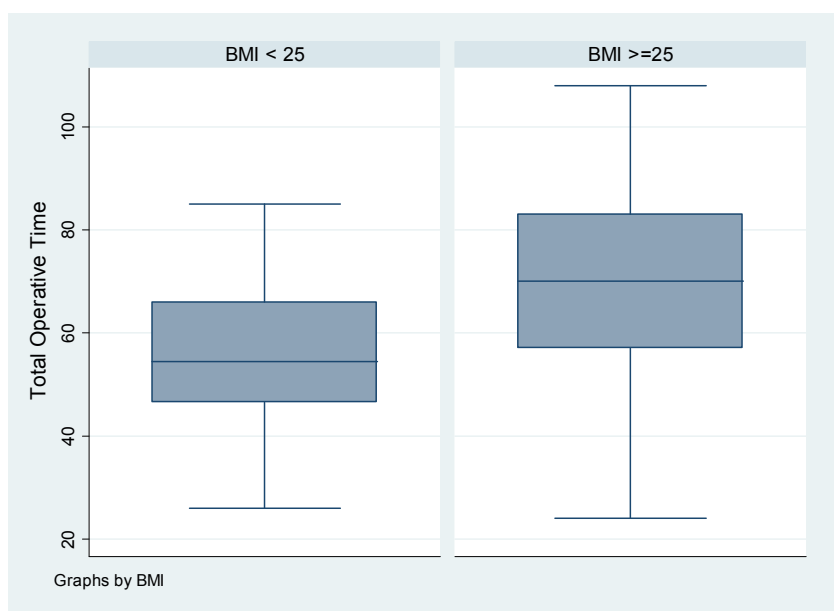

Figure 4. Variation on surgical time between patients with a $B M I<$ or $\geq 25$.

\section{Discussion}

In Day Surgery, we perform an average of ten surgical operations of unilateral inguinal hernia repair in two operating sessions every week. A single operative session lasts 7 hours, from 7 am to $2 \mathrm{pm}$. The mean time of each procedure is about 67 minutes and the turnover between two procedures is 10 minutes. Accordingly, five operations can be performed for each session, but many factors can influence surgical times and daily schedule. This inaccuracy in predicting case durations can result in three possible scenarios with obvious consequences on the operating room efficiency and on patient care: a) overutilization of Operating Room (OR); b) underutilization of OR, with excess staffing costs due to OR allocation not being based on maximizing OR Efficiency; c) one or more case cancellation, with consequent patient discomfort.

No classification is useful for preoperative planning considering surgical times. Indeed, while a novel direct hernia can be repaired in few minutes, a recurrent indirect inguinal hernia may require an hour.

From our study, we can highlight a statistically significant relationship between the surgical times and clinical parameters, such as the patient's sex, hernia reducibility, recurrent hernia and BMI. The classical distinction of inguinal hernias between direct and oblique was excluded from the criteria because clinical examination is often not sufficient to discriminate between the two different types of defect.

Based on these parameters, we can identify three patient groups, as shown in Table 3.

Table 3 The our new classification of patients in 3 groups, based on predictive clinical parameters of surgical times.

Table 3. Groups of patients based on the surgical schedule.

GROUP A (expected time $\leq 63$ minutes)

Female sex

Primitive hernia

Completely reducible hernia

$\mathrm{BMI}<25$

GROUP B (expected time 64-88 minutes)

Male sex 


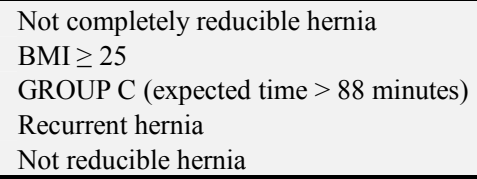

Conditions such as female sex, primitive and completely reducible hernia, BMI minor than 25 , lead to a surgical time equal to or less than 63 minutes (group A).

Male sex, not completely reducible hernia, and a BMI greater than 25 , determine time longer than the average time for the intervention of inguinal hernioplasty of 10-25 minutes (expected time 64-88 minutes - group B).

In patients with recurrent hernia or not reducible hernia (which constitutes $60 \%$ of the sample) we recorded average surgical time of more than 88 minutes (group C).

Estimated times are important for simulation models and for decision analysis based on such simulations.

Based on the aforementioned patient classification we will study a new OR organization, increasing efficiency.

Our results has been obtained by working with a fixed operating room team on consecutive similar cases as demonstrated by several studies $[17,18]$.

This experience can yield a new, not anatomical, classification of patients at the moment of the surgical visit hence facilitating the OR management where operating efficiency co-exists with the availability of time necessary and sufficient to perform the surgery "to perfection".

\section{Conclusions}

The aim of our study was to evaluate the operative times and consequently identify factors that affect the surgical time. As this study confirms, an optimal clinical patient evaluation should always be the first step to an effective organizational choice and it allows realistic predictions about the duration of inguinal hernioplasty. To know, also approximately, the duration of a surgical operation allows to reduce operating room costs and a minor time lose, ensuring more surgeries for each operative session.

This new classification can easily be adopted by every surgeon worldwide, ensuring a tailored operating session.

Further studies will be needed to enforce the effectiveness of this organizational model.

\section{Conflict of Interest}

The authors declare that they have no competing interests.

\section{References}

[1] Dabbas N, Adams K, Pearson K, Royle G. Frequency of abdominal wall hernias: is classical teaching out of date? JRSM Short Rep 2011; 2: 5 .
[2] The HerniaSurge Group. International guidelines for groin hernia management. Hernia. 2018 Feb; 22 (1): 1-165.

[3] Fitzgibbons RJ, Forse RA. Groin hernias in adults. N Engl J Med 2015; 372: 756-763.

[4] Jenkins JT et al. Inguinal hernias. BMJ. 2008 Feb 2; 336 (7638): 269-272.

[5] Kingsnorth A, LeBlanc K. Hernias: inguinal and incisional. Lancet. 2003 Nov 8; 362 (9395): 1561-71.

[6] AHRQ Pub. No. 12-EHC091-1 August 2012; Ambulatory Surgery Data From Hospitals and Ambulatory Surgery Centers: United States, 2010 - National Health Statistics Reports Number 102 February 28, 2017.

[7] Bay-Nielsen M, Kehlet H, Strand L, Malmstrom J, Andersen FH, Wara P, Juul P, Calleseen T. Quality assessment of 26, 304 herniorrhaphies in Denmark: a prospective nationwide study. Lancet. 2001 Oct 6; 358 (9288): 1124-8.

[8] Huerta S, Timmerman C, Argo M, Favela J, Pham T, Kukreja S, Yan J, Zhu H. Open, Laparoscopic, and Robotic Inguinal Hernia Repair: Outcomes and Predictors of Complications. J Surg Res. 2019 Apr 22; 241: 119-127.

[9] Marcy H. A new use of carbolized cat gut ligature. Boston Med Surg J. 1871; 85: 315-316.

[10] Amid PK, Shulman AG, Lichtenstein IL. Open "tension-free" repair of inguinal hernias: the Lichtenstein technique. Eur J Surg. 1996; 162: 447-453.

[11] Lichtenstein IL, Shulman AG, Amid PK, et al. The tensionfree hernioplasty. Am J Surg. 1989; 157: 188-193.

[12] Cooper A. The Anatomy and Surgical Treatment of Abdominal Hernia. London: Longman and Co.; 1804.

[13] Lichtenstein IL. Herniorrhaphy: a personal experience with 6321 cases. Am J Surg 1987; 153: 553-559.

[14] Zollinger RM Jr. A unified classification for inguinal hernias. Hernia 1999; 3: 195-200.

[15] Zollinger RM Jr. Classification of ventral and groin hernias. In: Fitzgibbons RJ Jr, Greenburg AG, editors. Nyhus and Condon's hernia. 5th edition. Philadelphia: Lippincott Williams \& Wilkins; 2002. p. 71-79.

[16] Matthews RD and Neumayer L. Inguinal hernia in the $21 \mathrm{st}$ century: an evidence-based review. Curr Probl Surg 2008; 45 (4): 261-312.

[17] Stepaniak PS et al. Working with a fixed operating room team on consecutive similar cases and the effect on case duration and turnover time. Arch Surg. 2010; 145 (12): 1165-1170.

[18] Friedman DM et al. Increasing operating room efficiency through parallel processing. Ann Surg. 2006; 243 (1): 10-14. 